Conduct i ve mi crobead ar ray det ecti on based on eddy-current test ing usi ng SV- GMR sensor and hel $\mathrm{mbl}$ ot $\mathrm{z}$ coil exciter

\begin{tabular}{|c|c|}
\hline 著者 & $\begin{array}{l}\text { Sonsak T., Chomsuwan K., Yamada Sot oshi, } \\
\text { I wahar a Nasayoshi }\end{array}$ \\
\hline $\begin{array}{l}\text { jour nal or } \\
\text { publ i cat i on title }\end{array}$ & $\begin{array}{l}\text { I NTERMAG } 2006 \text { - I EEE I nt er nat i onal Nagnet i cs } \\
\text { Conf er ence }\end{array}$ \\
\hline page $r$ ange & $3622-3624$ \\
\hline year & $2006-10-01$ \\
\hline URL & ht t p: //hdl . handl e. net /2297/11876 \\
\hline
\end{tabular}




\title{
Conductive Microbead Array Detection by High-Frequency Eddy-Current Testing Technique With SV-GMR Sensor
}

\author{
S. Yamada, Member, IEEE, K. Chomsuwan, Student Member, IEEE, T. Hagino, H. Tian, K. Minamide, and \\ M. Iwahara, Member, IEEE
}

Institute of Nature and Environmental Technology, Kanazawa University, Ishikawa 920-8667, Japan

\begin{abstract}
This paper describes the detection of conductive microbeads (PbSn) based on eddy-current testing (ECT) technique. High-frequency magnetic field applied to the conductive microbeads enables spin-valve giant magnetoresis-tance (SV-GMR) sensor to detect the magnetic fields occurred from eddy currents flowing in the conductive microbeads. In this paper, analysis of these magnetic fields by an analytical method is discussed and compared with experimental results.
\end{abstract}

Index Terms-Conductive microbead, eddy-current testing, high-frequency, spin-valve giant magnetoresistance.

\section{INTRODUCTION}

A PPLICATION of high-frequency eddy-current testing (ECT) probe with spin-valve type giant magnetoresistance (SV-GMR) sensor can be used to detect microdefects, such as the printed circuit board inspection [1]. Since SV-GMR sensor has many advantages, such as high-sensitivity to low magnetic field, high-spatial resolution, etc., it has been applied to various fields such as magnetic particle detection for bioengineering [2] and can also be used in the detection of the conductive microbeads ( $\mathrm{PbSn}$ ) by ECT technique.

Analysis of the magnetic field distribution created by the conductive microbeads by analytical method is proposed to study the parameters that effect the detection of the conductive microbeads. Experimentally, the detection results of the conductive microbeads with radius ranging from 125 to $300 \mu \mathrm{m}$ and of high-density conductive microbead array confirmed the feasibility of the proposed technique to recognize the conductive microbeads.

\section{Conductive Microbead Detection By EDDY-CURRENT TESTING}

\section{A. Structure of ECT Probe With SV-GMR Sensor}

The structure of the proposed ECT probe is shown in Fig. 1. The high-frequency exciting current is fed to the planar meander coil to generate a magnetic field only in $x$ and $y$ axis and to induce eddy currents flowing in the conductive microbeads. The SV-GMR sensor with effective areas of $193 \mu \mathrm{m} \times 180 \mu \mathrm{m}$ has pattern, as shown in Fig. 1, and was mounted on the meander coil in the opposite side of the conductive microbeads. The distance between the sensor and the conductive microbeads is around $162 \mu \mathrm{m}$. The sensing axis of the SV-GMR sensor was set to detect magnetic field in scanning direction.

Digital Object Identifier 10.1109/TMAG.2005.855172

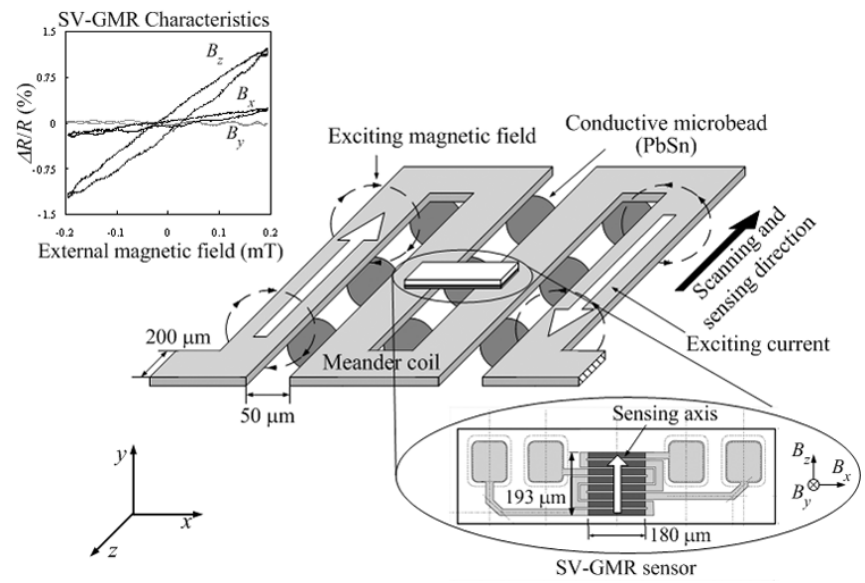

Fig. 1. ECT probe structure with conductive microbead $(\mathrm{PbSn})$ array and SV-GMR characteristics.

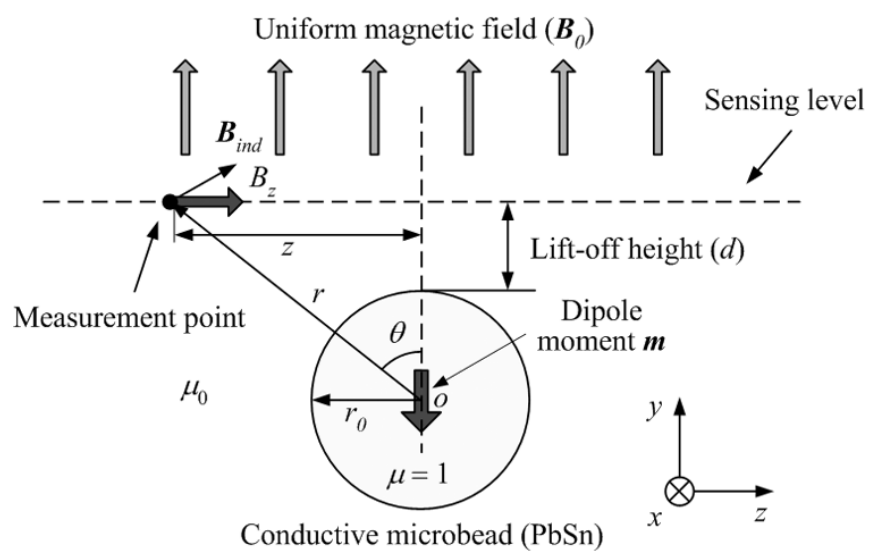

Fig. 2. Analytical model.

\section{B. Analysis of Microbead Magnetic Field by Analytical Method}

A simple model, as shown in Fig. 2, was used to analyze the magnetic field created by the conductive microbead $B_{z}$ on the sensing level. Assuming that the conductive microbead is 


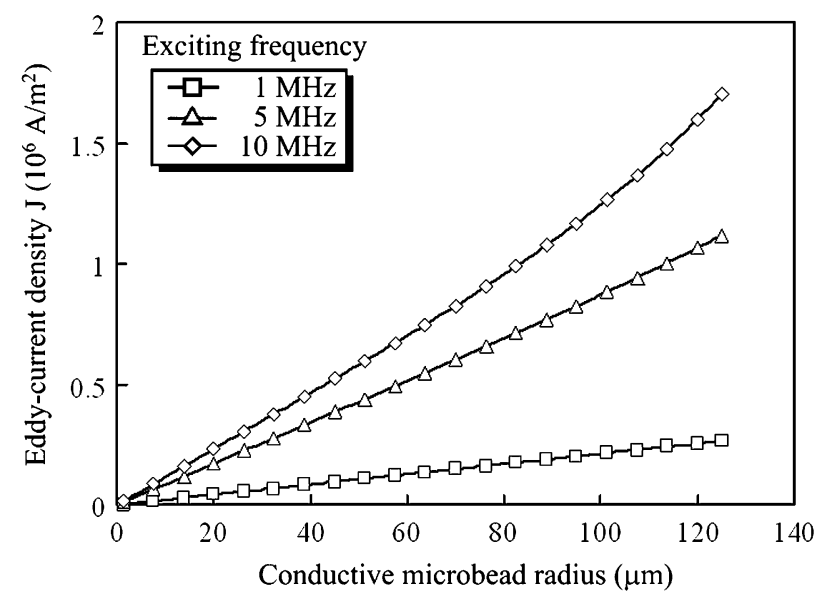

Fig. 3. Eddy-current density distribution in the conductive microbead $(\mathrm{PbSn})$ with $125 \mu \mathrm{m}$ radius and $162 \mu \mathrm{m}$ lift-off height.

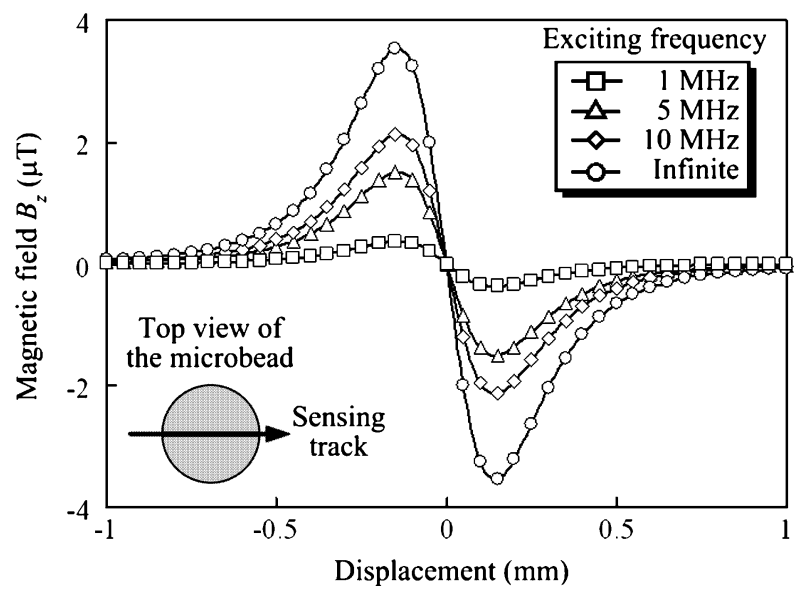

Fig. 4. Magnetic field $B_{z}$ over the sensing track obtained from analytical solution for a conductive microbead ( $\mathrm{PbSn}$ ) with $125 \mu \mathrm{m}$ radius and a lift-off height of $162 \mu \mathrm{m}$.

placed under uniform magnetic field $\boldsymbol{B}_{0}$, the eddy-current density flowing inside the microbead is expressed as

$$
J(r, \theta, \phi)=-j \omega \sigma a J_{1}(k r) B_{0} \sin \theta
$$

where

$$
\begin{array}{ll}
a & \frac{r_{0}}{\mu_{0}} \frac{1}{J_{1}\left(k r_{0}\right) / \mu_{0}+\left[k r_{0} J_{0}\left(k r_{0}\right)-J_{1}\left(k r_{0}\right)\right] / \mu} ; \\
k & (-1+j) \sqrt{\omega \sigma \mu / 2} ; \\
\sigma & \text { conductivity of the bead (PbSn: } \left.6.8 \times 10^{6} \mathrm{~S} / \mathrm{m}\right)
\end{array}
$$
$\mu_{0}$ and $\mu$ permeability of air and the microbead;

$J_{0}$ and $J_{1}$ zero and first order Bessel function [3].

From (1), the eddy-current density flowing in the conductive microbead is directly proportional with the frequency of the external magnetic field. Eddy-current density distributions in the conductive microbead with $125-\mu \mathrm{m}$ radius placed under the uniform magnetic fields $\boldsymbol{B}_{0}$ at $100 \mu \mathrm{T}$ are shown in Fig. 3 . Eddy-current density concentrates near the surface of the conductive microbead because of the skin depth effect.

The magnetic field density $B_{z}$ at measurement point on the sensing level is, therefore, expressed as

$$
B_{z}=3 b \frac{z\left(r_{0}+d\right)}{r^{5}} B_{0}
$$

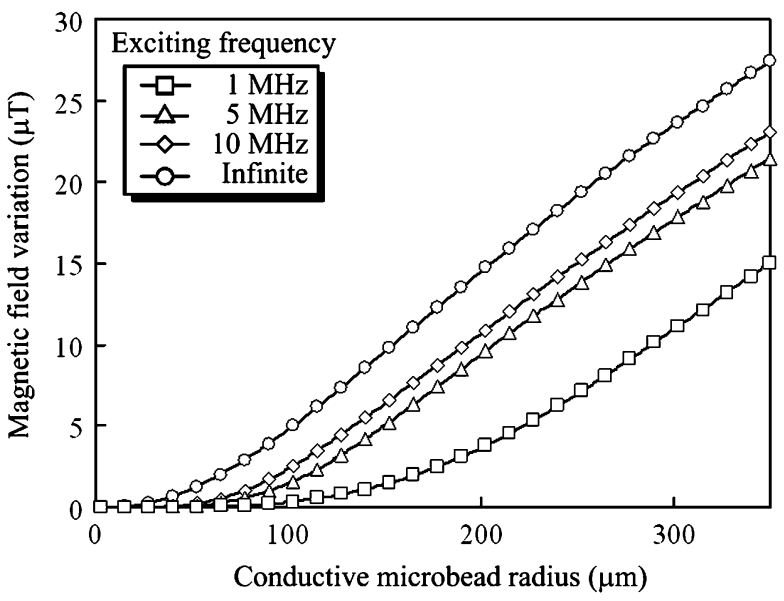

Fig. 5. Maximum magnetic field variation vs. conductive microbead $(\mathrm{PbSn})$ radius obtained from analytical solution where lift-off height is $162 \mu \mathrm{m}$.

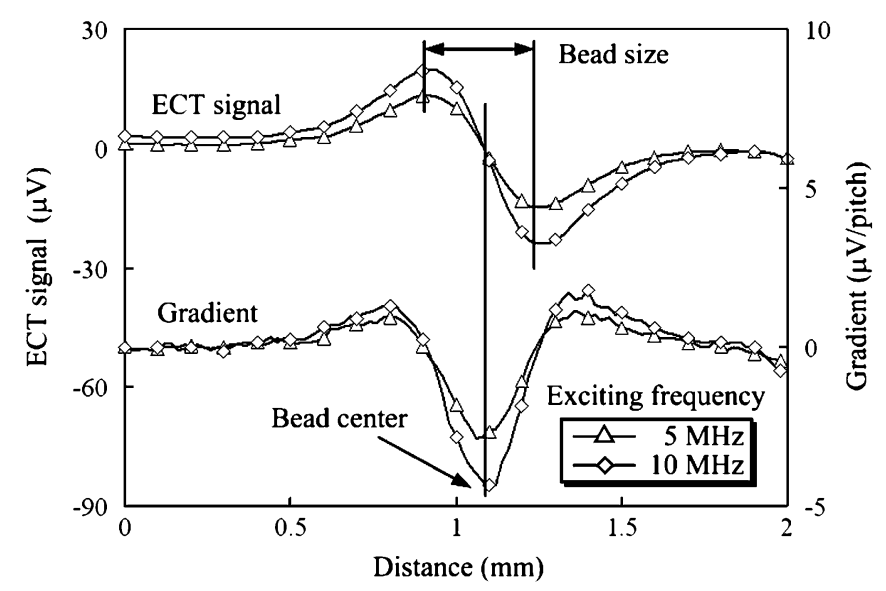

Fig. 6. ECT signal obtained from the detection of a conductive microbead $(\mathrm{PbSn})$ with $125 \mu \mathrm{m}$ radius and its signal gradient.

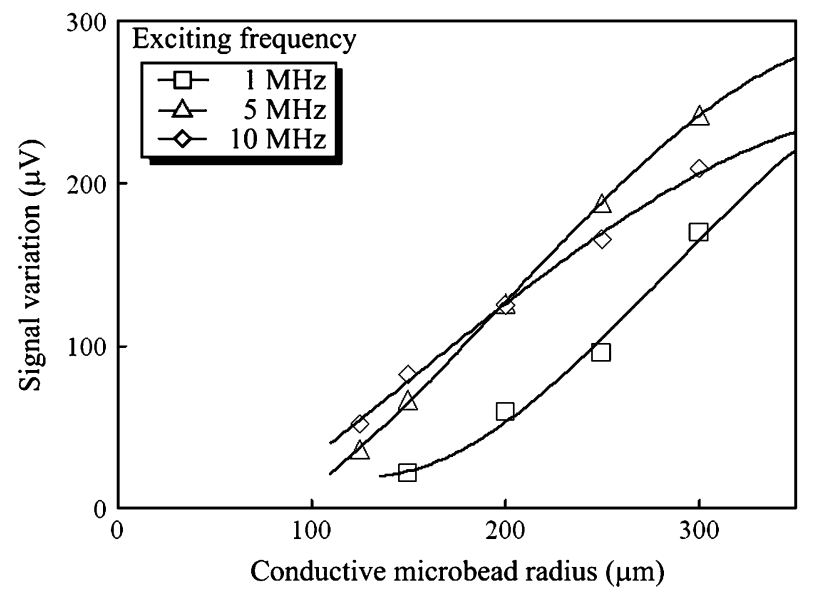

Fig. 7. Maximum signal variation vs. conductive microbead $(\mathrm{PbSn})$ radius obtained from the experiment.

where

$$
b=r_{0}^{3} \frac{J_{1}\left(k r_{0}\right) / 2 \mu_{0}-\left[k r_{0} J_{0}\left(k r_{0}\right)-J_{1}\left(k r_{0}\right)\right] / 2 \mu}{J_{1}\left(k r_{0}\right) / \mu_{0}+\left[k r_{0} J_{0}\left(k r_{0}\right)-J_{1}\left(k r_{0}\right)\right] / \mu} .
$$




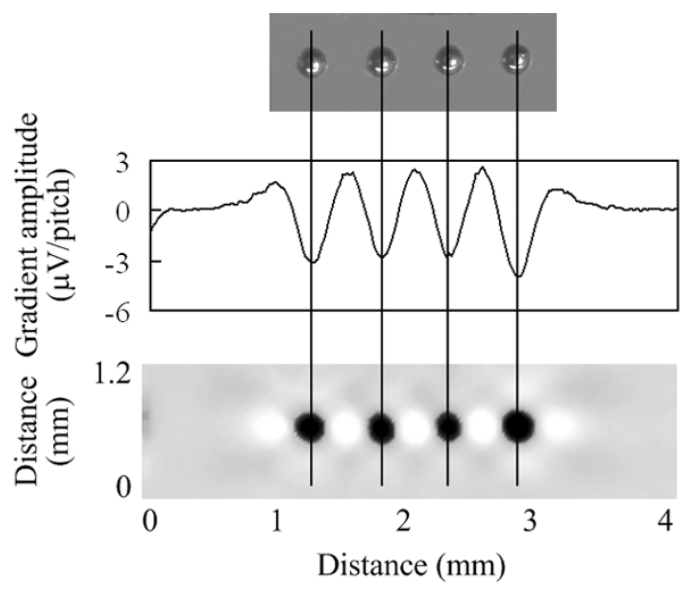

(a)

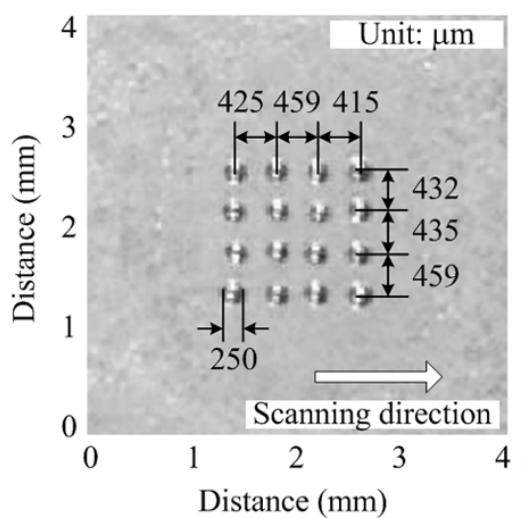

(b)

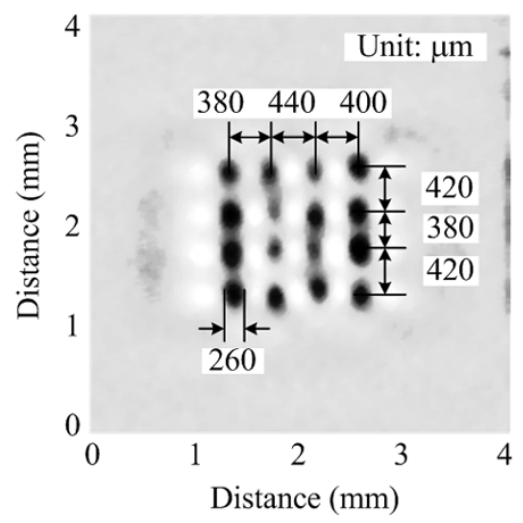

(c)

Fig. 8. Identification of the conductive microbead $(\mathrm{PbSn})$ position, conductive microbead $(\mathrm{PbSn})$ array model and its detection results. (a) Detection of bead position. (b) Conductive microbead array model. (c) Detection result.

When the exciting frequency approaches infinity, the magnetic field $B_{z}$ on the sensing level can be determined by magnetic dipole moment $\boldsymbol{m}$ that is derived as in (3) where $V$ is the volume of the conductive microbead.

$$
\boldsymbol{m}=-3 V \boldsymbol{B}_{0} / 2 \mu_{0} .
$$

Therefore, the magnetic field $B_{z}$ at measurement point when the exciting frequency approaches infinity is expressed as

$$
B_{z}=-\frac{9 V}{8 \pi} \frac{z\left(r_{0}+d\right)}{r^{5}} B_{0} .
$$

Figs. 4 and 5 show the frequency dependence of magnetic field variation obtained from eddy currents flowing in the conductive microbead placed under the uniform magnetic field $\boldsymbol{B}_{0}$ of $100 \mu \mathrm{T}$. The magnetic field varies at the position where the conductive microbead exists at the $0 \mathrm{~mm}$ displacement in Fig. 4. Peaks of magnetic field occur at the outside diameter of the conductive microbead. Moreover, the maximum amplitude of magnetic field variation is also depended on the conductive microbead radius, as shown in Fig. 5.

\section{Detection of Conductive Microbead}

\section{A. Characteristic of Conductive Microbead Detection}

The ECT signal waveforms in Fig. 6 obtained from the detection of a conductive microbead with $125-\mu \mathrm{m}$ radius at the frequency of 5 and $10 \mathrm{MHz}$ agree with the ECT signal waveforms obtained from analytical solution. The determinations of the microbead diameter and its position are done by considering the peak of ECT signal and peak of signal gradient, respectively. Fig. 7 shows the maximum variation of the ECT signal versus the radius of the conductive microbead, ranged from 125 to $300 \mu \mathrm{m}$. The maximum signal variation at exciting frequency of $10 \mathrm{MHz}$ decreases with the conductive microbead radius and it is lower than the signal variation at exciting frequency of $5 \mathrm{MHz}$ when the conductive microbead radius is bigger than $200 \mu \mathrm{m}$. This is because the planer meander coil can not generate the uniform magnetic field distribution. The experimental results also showed that signal variations at the conductive microbead depend on the frequency of the exciting magnetic field and the conductive microbead radius.

\section{B. Conductive Microbead Array Detection}

The application of numerical gradient technique to ECT signal enables us to easily determine the conductive microbead position as shown in Fig. 8(a). In addition, the pitches of the conductive microbead are also measured by considering the peak of the signal gradient. The conductive microbead array model with $250-\mu \mathrm{m}$ diameter and $410-460-\mu \mathrm{m}$ microbeads pitches and its detection results are shown in Figs. 8(b) and (c), respectively. The conductive microbeads are clearly recognized and the pitches of the conductive microbead are also accurately specified with error within $50 \mu \mathrm{m}$.

\section{CONCLUSION}

The analytical and experimental results showed that the ECT technique can be used to detect high-density conductive microbeads. The results also indicate that high-frequency ECT is useful and possible to use in physical measurement and biosensor applications.

\section{ACKNOWLEDGMENT}

This work was supported by the Japan Society for the Promotion of Science (Category B, 14350 218) under a Grand-in-Aid for Scientific Research.

\section{REFERENCES}

[1] S. Yamada, K. Chomsuwan, Y. Fukuda, M. Iwahara, H. Wakiwaka, and S. Shoji, "Eddy-Current testing probe with spin-valve type GMR sensor for printed circuit board inspection," IEEE Trans. Magn., vol. 40, no. 7, pp. 2676-2678, Jul. 2004.

[2] A. Anguelouch, D. H. Reich, C. L. Chien, and M. Tondra, "Detection of ferromagnetic nanowires using GMR sensors," IEEE Trans. Magn., vol. 40, no. 7, pp. 2997-2999, Jul. 2004.

[3] T. Morisue and M. Fukumi, "3-D eddy current calculation using magnetic vector potential," IEEE Trans. Magn., vol. 24, no. 1, pp. 106-109, Jan. 1988.

Manuscript received February 1, 2005 